\title{
Detección de necesidades de capacitación en docentes de la Universidad Virtual del Estado de Guanajuato
}

\section{Detection of training needs in teachers of the Virtual University of the State of Guanajuato}

\begin{abstract}
Rolando Granados Muñoz ${ }^{1} \&$ Wilehand Banda Sandoval
${ }^{1}$ Ingeniero en Gestión de Tecnologías de la Información con Maestría en Ciencias del Comportamiento. Especialista en Formación Docente de Educación Media Superior. https://orcid.org/0000-0002-8508-0112

La correspondencia en relación con este artículo debe dirigirse a Rolando Granados Muñoz, Departamento de Formación y Desarrollo Docente, Universidad Virtual del Estado de Guanajuato, Hermenegildo Bustos 129 A Sur, Colonia Centro, C.P. 36400, Purísima del Rincón, Guanajuato. Dirección electrónica: rogranados@uveg.edu.mx
\end{abstract}

\section{Dol https://doi.org/10.46589/rdiasf.vi35.382}

Recibido 13 de febrero 2021.

Aceptado 6 de junio 2021

Publicado 30 de junio de 2021

\section{Resumen}

La capacitación es un proceso esencial para el desarrollo y mejora continua de distintas instituciones, varias de las acciones que permiten este proceso de capacitación están antecedidas por distintas etapas, entre ellas se encuentra el Diagnóstico de Necesidades de Capacitación. Con la finalidad de realizar la detección de necesidades de capacitación, se llevó a cabo un estudio descriptivo con el objetivo de estudiar las necesidades de capacitación y las competencias de 838 docentes de educación media superior de la Universidad Virtual del Estado de Guanajuato. Para la recolección de los datos se diseñó un instrumento en formularios de Google compuesto por un cuestionario sociodemográfico, una escala tipo Likert, una sección de selección de cursos y una sección de respuesta abierta para la propuesta de cursos. Los resultados obtenidos del análisis de los datos efectuados a partir del SPSS 26 y Excel indican que existen diferencias significativas entre determinados grupos, las compasiones fueron realizadas entre mujeres y hombres (sexo), responsables y docentes (cargo) y el área de conocimientos (comunicación, ciencias exactas y 
naturales, ciencias sociales y humanidades); también se establecieron categorías de los cursos que solicitan los docentes. Los hallazgos encontrados en este estudio permiten corroborar los beneficios que conlleva la detección de necesidades de capacitación.

Palabras clave: Educación, enseñanza técnica y profesional, formación de docentes de secundaria, formación del personal docente.

\begin{abstract}
Training is an essential process for the development and continuous improvement of different institutions, several of the actions that allow this training process are preceded by different phases, among them is the Diagnosis of Training Needs. To establish the detection of training needs, a descriptive study was carried out with the objective of studying the training needs and competencies of 838 upper secondary education teachers of the Virtual University of the State of Guanajuato. To collect the data, an instrument was designed using Google forms consisting of a sociodemographic questionnaire, a Likert-type scale, a course selection section and an open response section for the course proposal. The results obtained from the analysis of the data carried out from SPSS 26 and Excel indicate that there are significant differences between certain groups, the comparisons were made between women and men (sex), managers and teachers (position) and the area of knowledge (communication, exact and natural sciences, social sciences and humanities); Categories of courses requested by teachers were also established. The findings found in this study allow corroborating the benefits of the detection of training needs.
\end{abstract}

\title{
Keywords
}

Education, educational personnel training, technical and vocational education, secondary teacher education. 


\section{Introducción}

Para las organizaciones la capacitación es una tarea fundamental para lograr un desarrollo fructífero y aprovechar el talento del personal que se encuentra colaborando activamente, el actualizar a sus miembros con nuevos conocimientos se vuelve crucial, pues mucho del éxito y logro de objetivos a ello se debe. En el ámbito educativo no deja de ser la excepción ya que se vuelve común llevar a cabo actividades de capacitación a través de distintas formas como pueden ser cursos, talleres, coloquios, asistencia a congresos, etc.

La capacitación es un proceso que implica planeación, sistematización, orden, programación y estrategias para que el grupo de personas a las que se dirija adquieran el conocimiento y habilidades necesarias para que sean desarrolladas y aplicadas principalmente en el área laboral. Estudios realizados en organizaciones muestran que existen aspectos positivos de todo este proceso de capacitación, estos aspectos van desde una valoración diagnóstica inicial hasta la implementación de un plan de capacitación elaborado a partir de las necesidades identificadas (Bermúdez, 2015; Cota \& Rivera, 2017; López, Reyes \& Molina, 2017; Puente, Acosta, Beltrán \& Torres, 2002). La capacitación forma parte de las instituciones y funciona acorde a sus estrategias, su calidad depende de un diseño específico que se adecue al perfil organizacional, también responde a los intereses de los trabajadores y se debe evaluar respecto a diversos indicadores que contribuyan al desarrollo del capital humano (Lay, Suaréz \& Samora, 2005; Ruíz, Baca \& Gutiérrez, 2011). Estas instituciones que usan la capacitación para la mejora constante de sus miembros llevan a cabo un proceso de varias etapas o en algunos casos fases, con la finalidad de buscar datos que permitan poner de manifiesto síntomas de situaciones problema, además de dar certeza que la capacitación es un medio y no un fin, es decir, que realmente representa una necesidad (Guiñazú, 2004; Méndez, 2002; Werther \& Davis, 2008). Cabe mencionar que este proceso en algunas ocasiones no está claramente definido, aunque se recomienda su aplicación de forma continua y de manera periódica en las organizaciones (Cruz \& Surdez, 2018).

Este proceso se denomina Diagnóstico de Necesidades de Capacitación o DNC; corresponde a la primera de las fases del proceso de capacitación y formación, ya que posterior a ello se elabora 
el plan de capacitación, se lleva a cabo su ejecución y se interpretan resultados, todo ello con el correspondiente diseño avalado con la rigurosidad científica y tecnológica (Méndez, 2004; Velázquez \& Peinado, 2010). Por su parte Sapién, Piñón y Gutiérrez (2014) agregan algunas fases importantes, entre ellas la identificación de los recursos y el control y seguimiento del mismo, además agregan el poco valor que se le da a los programas y planes de preparación sistemática.

En el DNC para la determinación de necesidades se utilizan diferentes tipos de herramientas e instrumentos, de acuerdo con Salas (2003) algunas de ellas son la entrevista, el cuestionario, la observación, la encuesta, reuniones de trabajo o la selección por parte del comité. Estas herramientas están relacionadas con las diferentes metodologías que se emplean para la detección de estas necesidades, la recomendación en educación es que sean empleados métodos mixtos (Herrera et al., 2013; Maya-Ampudia, Ángeles-Zavala \& Camarena-Olmedo, 2014). Incluso existen metodologías con propósitos particulares que funcionan no solamente para la detección de necesidades, sino para el perfeccionamiento docente a través de distintas dinámicas pedagógicas (Polo, 2003). Otras metodologías presentan una estructura más desarrollada, integrando cuatro etapas: la preparación, desarrollo, implementación y evaluación del impacto. Estas mismas etapas se componen por 11 fases y 15 pasos para el diagnóstico y proyección del sistema de formación y desarrollo por competencias (González-Verde \& Muñiz-Izquierdo, 2016). También se han desarrollado metodologías emergentes que agregan otros procesos, ejemplo de ello es la definición de cargos y niveles, así como la definición de perfiles ocupacionales antes del diagnóstico del estado actual de las necesidades; el otro proceso es la identificación de brechas antes de elaborar el plan de capacitación (Vergel, Parra \& Martínez, 2013).

Bajo esta perspectiva es que se manifiesta una estrecha relación entre la detección de necesidades como una fase inicial de entrenamiento con las competencias laborales orientadas al conocimiento (López \& Sarabia, 2016; Revilla, Acosta y Marval, 2009). De acuerdo con Rangel (2015) una competencia es "la capacidad del ser humano para realizar un conjunto de acciones, mediante la articulación de sus múltiples recursos personales (actitudes, conocimientos, emociones, habilidades, valores...), con el propósito de lograr una respuesta satisfactoria a un problema planteado en un contexto determinado" (p. 237).

ISSN: $2007-8870$

Universidad de Sonora
Los contenidos de este articulo extin bajo una licendia de Creative Commons Atribueión No Comercial - Sin Obra Derivada 4 , Ointernaciona : 
Cabe mencionar que las tendencias educativas contemplan competencias docentes afines a la gestión y procesos del aprendizaje, además de la comunicación y formación continua; y también involucra el desarrollo de las competencias profesionales, las cuales están relacionadas particularmente con actividades de investigación (Torres, Badillo, Valentin \& Ramírez, 2014). Un docente debe tener un perfil de habilidades mínimas para la práctica docente, para el proceso de enseñanza aprendizaje y para mejorar su desarrollo como profesor (Villarroel \& Bruna, 2017).

El perfil del docente se compone de seis dominios, de los cuales derivan los criterios e indicadores deseables para el docente en el ámbito escolar, la Secretaría de Educación Pública en México (2019) establece los siguientes:

a) Construye su identidad como docente de Educación Media Superior.

b) Comprende la normatividad de la Educación Media Superior que corresponde a la función docente.

c) Domina el currículo para la enseñanza y el aprendizaje.

d) Planifica e implementa los procesos de enseñanza y aprendizaje.

e) Colabora en el trabajo colegiado, de gestión y de vinculación del plantel.

f) Determina su trayectoria de formación, actualización y desarrollo profesional.

El sexto dominio refiere la responsabilidad que tiene el docente de Educación Media Superior para evaluar resultados de aprendizaje y las experiencias de sus pares con el objeto de generar un diagnóstico que permita la detección de necesidades de formación.

El análisis de su práctica docente debe realizarse pensando en el grado de desarrollo de las competencias docentes que establece el Acuerdo secretarial 477, en el que se integran ocho competencias profesionales (Secretaría de Educación Pública, 2008; Tejada, 2009):

1. Organiza su formación continua a lo largo de su trayectoria profesional.

2. Domina y estructura los saberes para facilitar experiencias de aprendizaje significativo. 
3. Planifica los procesos de enseñanza y de aprendizaje atendiendo al enfoque por competencias, y los ubica en contextos disciplinares, curriculares y sociales amplios.

4. Lleva a la práctica procesos de enseñanza y de aprendizaje de manera efectiva, creativa e innovadora a su contexto institucional.

5. Evalúa los procesos de enseñanza y de aprendizaje con un enfoque formativo.

6. Construye ambientes para el aprendizaje autónomo y colaborativo.

7. Contribuye a la generación de un ambiente que facilite el desarrollo sano e integral de los estudiantes.

8. Participa en los proyectos de mejora continua de su escuela y apoya la gestión institucional.

Desde una perspectiva similar Camargo-Escobar y Pardo-Adames (2008) proponen siete competencias básicas para el ejercicio de la función docente: planificación curricular, utilización adecuada de diseño metodológico y organización de actividades de enseñanza, competencia científica tecnológica, interacción adecuada con estudiantes, competencia para evaluar, competencia para realizar tutorías, autorreflexión sobre la práctica docente.

El desarrollo, fortalecimiento, identificación y evaluación de competencias docentes en los gestores del aprendizaje en las aulas favorece a una enseñanza que optimiza los recursos de los cuales se dispone en el sistema educativo, permitiendo impactar en situaciones problema u otros fenómenos sociales complejos de entender (Castro-Granados, Medina-Almeida \& Glasserman, 2017; Guzmán \& Marín, 2011). Estas competencias incluso en ambientes virtuales deben ser dominadas para la gestión estratégica del aprendizaje, el docente genera su alineación respecto a los criterios institucionales y las proyecciones que sean compartidas, ya sea que sus medios de enseñanza sean presenciales, en línea o mixtos (García et al., 2018; Guzmán, Marín, Zesati \& Breach, 2012).

Como se ha venido mencionando el rol de docente es primordial, ya lo mencionan Castillo, Yahuita y Garabito (2006), sus actividades lo posicionan para ser un transmisor de conocimientos, un animador, la persona que supervisa, un guía del aprendizaje o un investigador académico. 
Aparte de la función del docente, la participación del estudiante es fundamental, aunque sus visiones de aprendizaje y las actividades de evaluación suelen estar muy distantes, la comunicación y las relaciones que se desarrollen entre ambos es de suma importancia en el ambiente escolar (Martínez-Miguel, Solano, García-Carpintero \& Manso, 2018; Sáenz-Lozada, Cárdenas-Muñoz \& Rojas-Soto, 2010). Incluso Asún, Zúñiga y Ayala (2013) constatan que los estudiantes demandan un profesor con distintos tipos de habilidades que incluyen la planificación, organización, responsabilidad, dominio, etc., ello fija una alta expectativa que obliga al docente a desarrollar y fortalecer varios tipos de competencias.

Se ha revisado la importancia que tiene la Detección de Necesidades de Capacitación en los procesos de formación docente y, sobre todo, en la identificación de competencias docentes. En esa directiva, se planteó como objetivo estudiar las necesidades de capacitación y las competencias docentes de educación media superior en la Universidad Virtual del Estado de Guanajuato.

\section{Material y método}

\section{Participantes}

En el estudio participaron 838 docentes de educación media superior de la Universidad Virtual del Estado de Guanajuato. La distribución por sexo indica la participación del 59\% mujeres y el $41 \%$ hombres. El 64\% indicó tener un rol de docente y el 36\% de responsable del centro de trabajo. De esta muestra 282 fueron del área de comunicación, 272 del área de ciencias exactas y experimentales y finalmente 284 del área de ciencias sociales y humanidades.

\section{Instrumento}

Se aplicó un cuestionario sociodemográfico a través de un formulario Google, en este se especificaba la dirección de correo electrónico, nombre completo del docente, número telefónico, área de conocimiento, cargo (rol), sexo y el centro de trabajo.

Se diseñó una escala tipo Likert de cuatro puntos con dos opciones de respuesta, un tipo de respuesta descriptiva de intensidad (bajo, regular, alto, muy alto) y otro de tipo de respuesta de 
frecuencia (algunas veces, muy a menudo, normalmente, siempre). La escala cuenta con un total de 28 ítems distribuidos en ocho dimensiones, estas dimensiones son las competencias docentes que establece el acuerdo secretarial 447.

Se agregaron dos secciones de selección de cursos, la primera sección agrupó la dimensión integral, la dimensión digital y la dimensión profesional, cada una con cuatro opciones de selección de curso. La segunda sección fue de respuesta abierta, un ítem que pedía un curso de prioridad 1 y otro ítem igual que pedía un curso de prioridad 2.

\section{Procedimiento}

Una vez diseñado el instrumento ad hoc para la detección de necesidades de capacitación, como un procedimiento del Departamento de Formación y Desarrollo Docente se envió el formulario a través de correo electrónico al personal docente de Telebachillerato Comunitario de la Universidad Virtual del Estado de Guanajuato. El instrumento estuvo a disposición durante cinco días hábiles para su respuesta y se llevó a cabo un seguimiento intermedio a través de un recordatorio y un monitoreo constante a dudas y respuestas. A los docentes se les comunicó que la información que fuera aportada sería manejada de manera confidencial.

Los datos fueron trabajados a través de la estadística descriptiva e inferencial, haciendo uso de herramientas informáticas como Excel y el SPSS 26. Las pruebas estadísticas aplicadas para el análisis de la información sociodemográfica fueron las tablas cruzadas (crosstabs). Para el análisis de las escalas se generaron variables promedio de los ítems que conforman cada una de las competencias, al final resultaron 8 competencias que fueron las que se compararon con la prueba $t$ de student para muestras independientes, estas comparaciones se hicieron para saber las diferencias significativas entre los grupos de sexo (mujer y hombre) y cargo (responsable y docente). La ANOVA de un factor con el respectivo post hoc HSD de Tukey se aplicó para saber las diferencias significativas entre las comparaciones de los grupos por área de conocimiento (Comunicación; Ciencias Exactas y Experimentales; Ciencias Sociales y Humanidades). 
En el análisis de selección de cursos las frecuencias se trabajaron mediante Excel y para los ítems de opción de respuesta abierta se procesaron los datos a través de la categorización de los cursos.

\section{Resultados}

De la muestra total se obtiene una mayor participación de mujeres que de hombres; solamente en el área de Ciencias Exactas y Experimentales los hombres superan a las mujeres. En la Tabla 1 se puede apreciar la proporción por sexo en cada una de las áreas de conocimiento, se destaca una mayor participación de mujeres del área de Ciencias Sociales y Humanidades seguido de las mujeres del área de Comunicación.

\section{Tabla 1}

\section{Distribución del sexo del docente por área de conocimiento}

\begin{tabular}{|c|c|c|c|c|c|}
\hline \multirow{2}{*}{ Sexo } & \multirow{2}{*}{ Descripción } & \multicolumn{3}{|c|}{ Area de conocimiento } & \multirow{2}{*}{ Total } \\
\hline & & COM & CEyE & CSyH & \\
\hline \multirow{4}{*}{ Mujer } & & 181 & 125 & 184 & 490 \\
\hline & $\%$ dentro de género & $36.9 \%$ & $25.5 \%$ & $37.6 \%$ & $100.0 \%$ \\
\hline & $\begin{array}{c}\% \text { dentro de Area de } \\
\text { conocimiento }\end{array}$ & $64.2 \%$ & $46.0 \%$ & $64.8 \%$ & $58.5 \%$ \\
\hline & $\%$ del total & $21.6 \%$ & $14,9 \%$ & $22.0 \%$ & $58.5 \%$ \\
\hline \multirow{4}{*}{ Hombre } & & 101 & 147 & 100 & 348 \\
\hline & $\%$ dentro de género & $29.0 \%$ & $42.2 \%$ & $28.7 \%$ & $100.0 \%$ \\
\hline & $\begin{array}{c}\% \text { dentro de Area de } \\
\text { conocimiento }\end{array}$ & $35.8 \%$ & $54.0 \%$ & $35.2 \%$ & $41.5 \%$ \\
\hline & $\%$ del total & $12.1 \%$ & $17.5 \%$ & $11.9 \%$ & $41.5 \%$ \\
\hline
\end{tabular}

Fuente: Elaboración propia.

Otra de las características de la muestra es que la mayor parte de los responsables que participaron pertenecen al área de Comunicación que en su mayoría son mujeres; como lo permite observar la Tabla 2, el área de Ciencias Exactas y Experimentales y el área de Ciencias Sociales y Humanidades mostraron una tendencia más proporcional de participación con personas con un cargo de docente. 
Tabla 2

Distribución de cargo del docente por área de conocimiento

\begin{tabular}{|c|c|c|c|c|c|}
\hline \multirow{2}{*}{ Cargo } & \multirow{2}{*}{ Descripción } & \multicolumn{3}{|c|}{ Área de conocimiento } & \multirow{2}{*}{ Total } \\
\hline & & COM & CEyE & CSyH & \\
\hline \multirow{4}{*}{ Responsable } & & 117 & 91 & 94 & 302 \\
\hline & $\%$ dentro de cargo & $38.7 \%$ & $30.1 \%$ & $31.1 \%$ & $100.0 \%$ \\
\hline & $\begin{array}{l}\% \text { dentro de Area de } \\
\text { conocimiento }\end{array}$ & $41.5 \%$ & $33.5 \%$ & $33.1 \%$ & $36.0 \%$ \\
\hline & $\%$ del total & $14.0 \%$ & $10.9 \%$ & $11.2 \%$ & $36.0 \%$ \\
\hline \multirow{4}{*}{ Docente } & & 165 & 181 & 190 & 536 \\
\hline & $\%$ dentro de cargo & $30.8 \%$ & $33.8 \%$ & $35.4 \%$ & $100.0 \%$ \\
\hline & $\begin{array}{l}\% \text { dentro de Arrea de } \\
\text { conocimiento }\end{array}$ & $58.5 \%$ & $66.5 \%$ & $66.9 \%$ & $64.0 \%$ \\
\hline & $\%$ del total & $19.7 \%$ & $21.6 \%$ & $22.7 \%$ & $64.0 \%$ \\
\hline
\end{tabular}

Fuente: Elaboración propia.

En el análisis inferencial de la autoevaluación de competencias los resultados indican que los docentes en todas las competencias refieren estar arriba de la media teórica que es de dos, considerando la escala Likert de cuatro puntos. La competencia que puntúan menor es la 5 (Evalúa los procesos de enseñanza y de aprendizaje con un enfoque formativo) y la competencia docente que consideran más fortalecida es la 7 (Contribuye a la generación de un ambiente que facilite el desarrollo sano e integral de los estudiantes). 
Como se aprecia en la Tabla 3 los hombres puntúan arriba de las mujeres en la competencia 2 (Domina y estructura los saberes para facilitar experiencias de aprendizaje significativo). Por el contrario, en la competencia 5 (Evalúa los procesos de enseñanza y de aprendizaje con un enfoque formativo) y en la competencia 8 (Participa en los proyectos de mejora continua de su escuela y apoya la gestión institucional) las mujeres puntúan por arriba de los hombres.

\section{Tabla 3}

Diferencias significativas en las competencias docentes por sexo

\begin{tabular}{|c|c|c|c|c|c|c|c|}
\hline $\begin{array}{l}\text { Competencia } \\
\text { docente }\end{array}$ & Sexo & Media & $\begin{array}{c}\text { Desviación } \\
\text { estàndar }\end{array}$ & $\begin{array}{c}\text { t de } \\
\text { student }\end{array}$ & 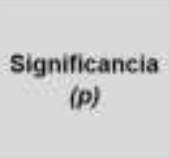 & $\begin{array}{l}\text { Intervalo } \\
\text { de } \\
\text { confianza } \\
\text { inferior }\end{array}$ & $\begin{array}{c}\text { Intervalo } \\
\text { de } \\
\text { confianza } \\
\text { superior }\end{array}$ \\
\hline \multirow{2}{*}{$\mathrm{CD} 2$} & Mujer & 2.8122 & .51208 & \multirow{2}{*}{-2.61} & \multirow{2}{*}{.009} & \multirow{2}{*}{-.165} & \multirow{2}{*}{-.023} \\
\hline & Hombre & 2.9066 & .52107 & & & & \\
\hline \multirow{2}{*}{ CD5 } & Mujer & 2.8034 & .56933 & \multirow{2}{*}{2.187} & \multirow{2}{*}{.029} & \multirow{2}{*}{.009} & \multirow{2}{*}{.165} \\
\hline & Hombre & 2.7165 & .56366 & & & & \\
\hline \multirow{2}{*}{ CD8 } & Mujer & 2.8709 & .62767 & \multirow{2}{*}{2.032} & \multirow{2}{*}{.042} & \multirow{2}{*}{.003} & \multirow{2}{*}{.174} \\
\hline & Hombre & 2.7823 & .61343 & & & & \\
\hline
\end{tabular}

Fuente: Elaboración propia.

En la autoevaluación realizada por los participantes, los resultados se muestran en la Tabla 4, en este caso los responsables de los centros de Telebachillerato Comunitario puntúan mayor que los docentes en la competencia 1 (Evalúa los procesos de enseñanza y de aprendizaje con un enfoque formativo), en la competencia 3 (Planifica los procesos de enseñanza y de aprendizaje atendiendo al enfoque por competencias, y los ubica en contextos disciplinares, curriculares y sociales amplios), en la competencia 7 (Contribuye a la generación de un ambiente que facilite el 
desarrollo sano e integral de los estudiantes) y en la competencia 8 (Participa en los proyectos de mejora continua de su escuela y apoya la gestión institucional).

\section{Tabla 4}

\section{Diferencias significativas en las competencias docentes por el cargo que ocupan}

\begin{tabular}{|c|c|c|c|c|c|c|c|}
\hline $\begin{array}{l}\text { Competencia } \\
\text { docente }\end{array}$ & Cargo & Media & $\begin{array}{l}\text { Desviación } \\
\text { estándar }\end{array}$ & $\begin{array}{c}\text { t de } \\
\text { student }\end{array}$ & $\begin{array}{c}\text { Stgnificancla } \\
(p)\end{array}$ & $\begin{array}{c}\text { Intervalo } \\
\text { de } \\
\text { confianza } \\
\text { inferior }\end{array}$ & 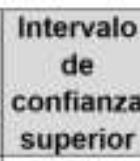 \\
\hline \multirow{2}{*}{ CD1 } & Responsable & 3.3226 & .48364 & \multirow{2}{*}{3.051} & \multirow{2}{*}{.002} & \multirow{2}{*}{.040} & \multirow{2}{*}{.185} \\
\hline & Docente & 3.2100 & .52855 & & & & \\
\hline \multirow{2}{*}{ CD3 } & Responsable & 2.9216 & .73822 & \multirow{2}{*}{2.25} & \multirow{2}{*}{.025} & \multirow{2}{*}{.015} & \multirow{2}{*}{.227} \\
\hline & Docente & 2.8004 & .75501 & & & & \\
\hline \multirow{2}{*}{ CD7 } & Responsable & 3.4888 & .48560 & \multirow{2}{*}{2.438} & \multirow{2}{*}{.015} & \multirow{2}{*}{.017} & \multirow{2}{*}{.163} \\
\hline & Docente & 3.3986 & .53027 & & & & \\
\hline \multirow{2}{*}{ CD8 } & Responsable & 2.8965 & .61832 & \multirow{2}{*}{2.181} & \multirow{2}{*}{.029} & \multirow{2}{*}{.009} & \multirow{2}{*}{.185} \\
\hline & Docente & 2.7990 & .62338 & & & & \\
\hline
\end{tabular}

Fuente: Elaboración propia.

Los resultados obtenidos a través del análisis ANOVA revelan que los docentes de Ciencias Exactas y Experimentales refieren una mayor puntuación que los docentes de las otras áreas de conocimiento en la competencia 2 (Domina y estructura los saberes para facilitar experiencias de aprendizaje significativo); los docentes de Comunicación refieren una mayor 
puntuación que los docentes de Ciencias Exactas y Experimentales en la competencia 5 (Evalúa los procesos de enseñanza y de aprendizaje con un enfoque formativo) y en la competencia 8 (Participa en los proyectos de mejora continua de su escuela y apoya la gestión institucional). Estas aseveraciones se sustentan en los datos estadísticos que se presentan en la Tabla 5.

\section{Tabla 5}

Diferencias significativas en las competencias docentes por área de conocimiento

\begin{tabular}{|c|c|c|c|c|}
\hline $\begin{array}{c}\text { Competencia } \\
\text { docente }\end{array}$ & $\begin{array}{c}\text { Area de } \\
\text { conocimiento }\end{array}$ & $\begin{array}{c}\text { Significancia } \\
(p)\end{array}$ & Media & $\begin{array}{l}\text { Significancia del } \\
\text { modelo }\end{array}$ \\
\hline \multirow{3}{*}{$\mathrm{CD} 2$} & $\mathrm{COM}$ & .006 & 2.8174 & \multirow{3}{*}{$(F=5.69, d f=2, p=.004)$} \\
\hline & CEyE & & 2.9375 & \\
\hline & $\mathrm{CSyH}$ & .017 & 2.8028 & \\
\hline \multirow{2}{*}{ CD5 } & $\mathrm{COM}$ & \multirow{2}{*}{.006} & 2.8322 & \multirow{2}{*}{$(F=4.82, d f=2, p=.008)$} \\
\hline & CEyE & & 2.6850 & \\
\hline \multirow{2}{*}{$\mathrm{CD} 8$} & COM & \multirow{2}{*}{.004} & 2.9034 & \multirow{2}{*}{$(F=5.39, d f=2, p=.005)$} \\
\hline & CEyE & & 2.7362 & \\
\hline
\end{tabular}

Fuente: Elaboración propia.

Los resultados de la selección de cursos arrojan las frecuencias que se presentan a continuación; los cursos a elegir se encontraban divididos en tres dimensiones:

1. Dimensión integral

a. Creatividad (553 veces en selección).

b. Neurolingüística (424 veces en selección). 
c. Resiliencia, positivismo y manejo de emociones (364 veces en selección).

d. Motivación (282 veces en selección)

2. Dimensión digital
a. Portafolios digitales (478 veces en selección)
b. Evaluación digital (469 veces en selección)
c. Almacenamiento (335 veces en selección)
d. Uso de redes sociales (298 veces en selección)
e.

3. Dimensión profesional
a. Estrategias de enseñanza (709 veces en selección)
b. Planeaciones (358 veces en selección)
c. Socioformación (302 veces en selección)
d. Evaluación (245 veces en selección)

En el análisis de respuesta abierta los docentes debían proporcionar dos cursos, un curso de prioridad 1 y otro de prioridad 2; resultaron 11 categorías oscilando entre las 25 y las 90 menciones como se puede apreciar en la Figura 1.

\section{Figura 1}

\section{Categorías de los cursos mencionados con prioridad 1 y prioridad 2}




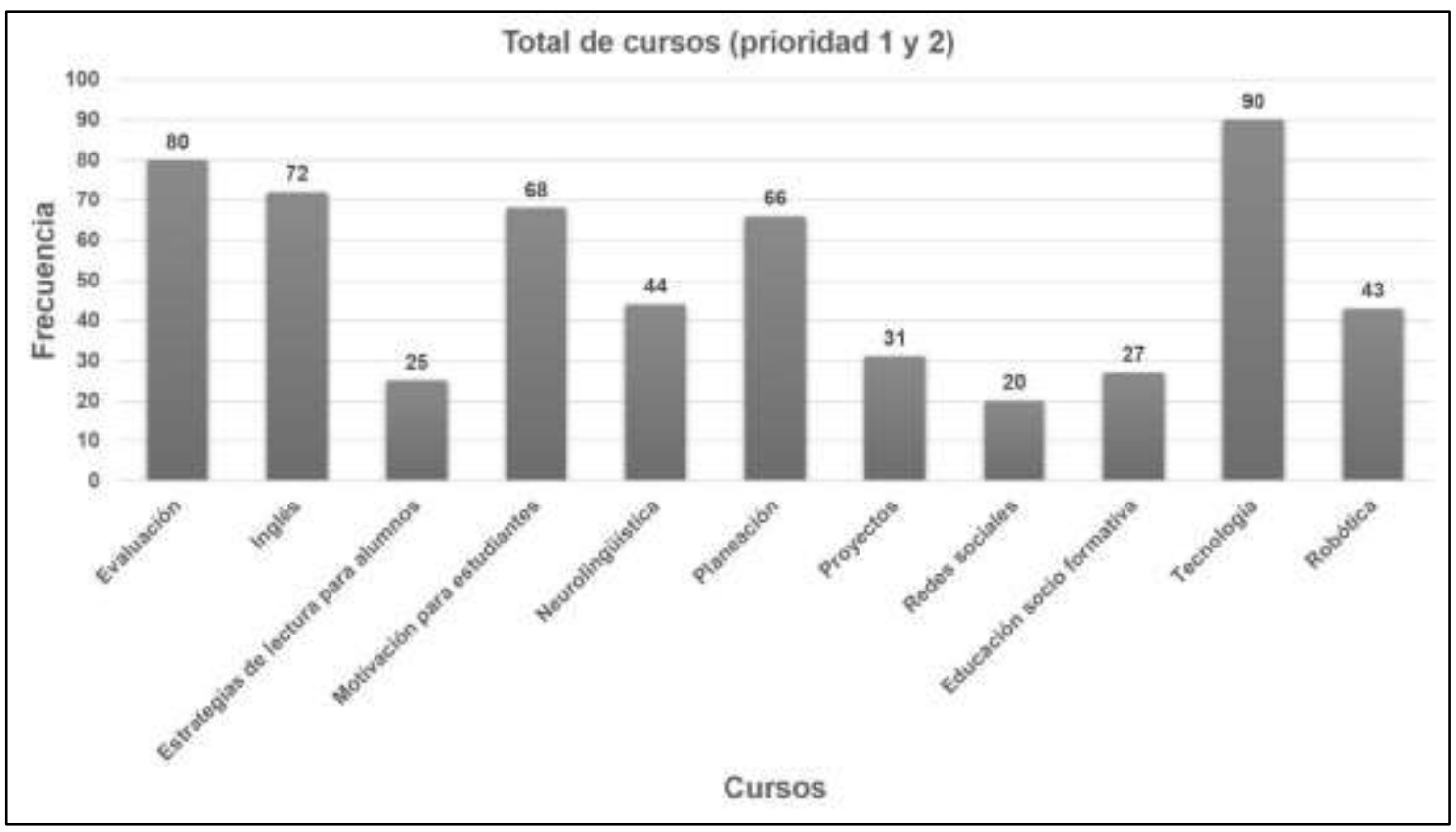

Fuente: Elaboración propia.

Las categorías presentadas están compuestas por las solicitudes de cursos que realizaron los docentes, algunos de los cursos más representativos son los siguientes:

1. Evaluación: elaboración de instrumentos de evaluación, evaluación formativa y sumativa a estudiantes, evaluación mediante exámenes en línea, productos e indicadores de evaluación, diagnóstico de problemas de aprendizaje.

2. Inglés: estrategias de enseñanza aprendizaje para el idioma inglés, estrategias para la asignatura de inglés, reforzamiento del inglés.

3. Estrategias de lectura para alumnos: comprensión lectora para aplicar a los alumnos, cómo fomentar la lectura en los estudiantes, lectura de comprensión y redacción de textos, taller de lectura y redacción.

4. Motivación para estudiantes: motivar a los estudiantes a continuar sus estudios, cómo motivar a los estudiantes, cómo generar estrategias de permanencia escolar, motivación a estudiantes en riesgo de abandono, motivación y manejo de emociones en alumnos. 
5. Neurolingüística: neurolingüística en el aula, neurolingüística aplicada en la educación, lenguaje neurolingüístico, programación neurolingüística.

6. Planeación: planeaciones óptimas, realizar planeaciones para aplicarlas a distancia, estrategias de planeación y secuencia didáctica, mejoramiento de planeación y secuencias didáctica.

7. Proyectos: proyectos de trabajo colaborativo, diseño y desarrollo de proyectos de investigación, gestión de proyectos, desarrollo de proyectos productivos, proyectos interdisciplinarios, cómo realizar proyectos de investigación escolares.

8. Redes sociales: estrategias de aprendizaje mediante la aplicación WhatsApp, el buen uso de la tecnología y redes sociales en los jóvenes, WhatsApp en el uso docente, uso adecuado de redes sociales.

9. Educación socio formativa: enfoque socioformativo, educación socioformativa, habilidades socioformativas, emociones socioformativas.

10. Tecnología: uso de las herramientas tecnológicas profesionales para docentes, uso de aplicaciones para laboratorios virtuales, almacenamiento de información, uso de medios electrónicos para la educación, uso de la nube para las evidencias, material didáctico digital.

11. Robótica: aplicación de ciencias experimentales con uso de robótica, robótica educativa, programación del equipo de robótica, programación de robots.

La distribución de frecuencias en estas categorías marca una tendencia de los docentes del área de conocimiento de Comunicación pidiendo cursos dentro de la categoría de inglés como prioridad 1 y como prioridad 2. Los docentes del área de conocimientos de Ciencias Exactas y Experimentales piden como prioridad 1 cursos que forman parte de la categoría de robótica y como prioridad 2 cursos dentro de la categoría de evaluación. Los docentes del área de conocimientos de Ciencias Sociales y Humanidades piden como prioridad 1 cursos que forman parte de la categoría de planeación y como prioridad 2 cursos dentro de la categoría de evaluación y de la categoría de tecnología. 


\section{Discusión}

En lo extenso de este trabajo se hicieron distintas menciones acerca de la importancia del proceso de capacitación para el cumplimiento de metas. La adquisición de conocimiento y habilidades para cumplir mejor con los intereses institucionales se vuelve una tarea fundamental. En un inicio se hizo énfasis de los aspectos positivos de la capacitación, así como sus implicaciones dentro de las instituciones en función de sus objetivos, este proceso resulta ser de gran trascendencia (Bermúdez, 2015; Cota \& Rivera, 2017; Lay, Suaréz \& Samora, 2005; López, Reyes \& Molina, 2017; Puente, Acosta, Beltrán \& Torres, 2002; Ruíz, Baca \& Gutiérrez, 2011). En ese acontecer es que la Universidad Virtual de Estado de Guanajuato a través del Departamento de Formación y Desarrollo Docente tiene como misión ser un área que atienda las demandas de capacitación docente de una manera eficiente. Como lo sugiere la teoría (Cruz \& Surdez, 2018; Guiñazú, 2004; Méndez, 2002; Werther \& Davis, 2008) estas actividades de capacitación se realizan de manera continua y periódica procurando detectar incidentes que puedan resultar potencialmente problemas, además de asegurarse de estar dando un servicio acorde a los requerimientos del personal docente.

La detección de necesidades de capacitación o también denominado Diagnóstico de Necesidades de Capacitación (DNC) representa una opción viable para elaborar planes o programas que incluyan los cursos, talleres, seminarios, conferencias, etc., destinados a cumplir con las expectativas de los participantes que los solicitan. Esta detección de necesidades no es una labor simple, y aunque pudiera considerarse solamente una práctica institucional más, según algunos teóricos (Méndez, 2004; Velázquez \& Peinado, 2010) la obtención, interpretación y manejo de los datos obtenidos deben ser trabajados con la calidad que obliga cualquier estudio científico. Las diferentes herramientas e instrumentos para la recolección de información deben adecuarse a los objetivos y a la muestra que se está estudiando, es por ello que las metodologías a emplear son variadas y permiten cumplir a cabalidad con propósitos particulares, ya sea que desarrollen una estructura propia o que sumen procesos, fases o etapas (González-Verde \& Muñiz-Izquierdo, 2016; 
Herrera et al., 2013; Maya-Ampudia, Ángeles-Zavala \& Camarena-Olmedo, 2014; Polo, 2003; Salas, 2003; Vergel, Parra \& Martínez, 2013).

Como ya se mencionó con anterioridad, la detección de necesidades de capacitación es solamente parte inicial de un proceso que permite favorecer al fortalecimiento y desarrollo de competencias (López \& Sarabia, 2016; Revilla, Acosta y Marval, 2009). Estas competencias deben forjarse a partir de habilidades que configuran el perfil de un docente (Villarroel \& Bruna, 2017); es por ello que la Secretaría de Educación Pública en México (2019) establece seis dominios que configuran este perfil en la docencia de educación media superior y que norman de manera formal las prácticas educativas atendiendo a la necesidad de reforzar ocho competencias docentes (Secretaría de Educación Pública, 2008; Tejada, 2009), mismas que fueron la pauta para la elaboración del presente estudio; en este estudio se encontraron hallazgos importantes, por ejemplo las diferencias que existen entre mujeres y hombres, entre responsables y docentes, o bien, entre las áreas de conocimiento, esto permite conocer que existen competencias docentes en determinados grupos que deben ser prioridad al momento de lanzar las capacitaciones.

Algo que vale la pena hacer evidente es que estas competencias contienen ítems de los cuales no se tiene demostrada su validez y confiabilidad con investigaciones anteriores; esta es una limitación importante y que en próximas ediciones en la detección de necesidades se debe mejorar, hacer el diagnóstico con un instrumento como el que presenta Camargo-Escobar y Pardo-Adames (2008) basado en siete competencias básicas para el ejercicio de la función docente es medular al menos para sumar a la calidad y cientificidad de los datos. En esta investigación, aunque no se realizaron procedimientos previos como algún jueceo, plan de prueba o discriminación de ítems, etc., su diseño se hizo a consciencia por personal del Departamento de Formación y Desarrollo Docente tomando como estructura teórica las competencias docentes del Acuerdo secretarial 447.

Se hizo inferencia en su momento de la importancia de la identificación y evaluación de competencias docentes para generar impactos en situaciones problema o fenómenos sociales que sumen al aprendizaje y que apoyen a la misión y a los objetivos de las instituciones que las emplean (Castro-Granados, Medina-Almeida \& Glasserman, 2017; García et al., 2018; Guzmán \& Marín, 
2011; Guzmán, Marín, Zesati \& Breach, 2012). Incluso esto es perceptible en los resultados obtenidos en este estudio, los cursos que tuvieron que seleccionar los docentes se relacionan con el contexto de sus labores, y sobre todo hace accesible la posibilidad de adaptar distintos tipos de técnicas a las situaciones del docente y del alumnado. Un aspecto importante que se destaca es la categorización de los cursos ya que permitió acentuar esta idea, los cursos que se dieron a la tarea de proponer los docentes no distan mucho de los que se les mostraron para selección, sin embargo, se identificó que los docentes, aunque pidan cursos similares, su petición se enfoca en sumar en el conocimiento de las técnicas para poder enseñar de manera eficiente los temas, un ejemplo es que no solicitan un curso de inglés, sino cómo enseñar estratégicamente el inglés. Bien lo establece la teoría (Asún, Zúñiga \& Ayala, 2013; Castillo, Yahuita \& Garabito, 2006), el docente puede desempeñar varios roles, pero, ante todo, los estudiantes le demandan habilidades que lo obligan al desarrollo y fortalecimiento de competencias.

En términos del DNC aquí expuesto se puede precisar que para las instituciones no solamente debe representar una obligación, sino que se vuelve una tarea fundamental que exige ir más allá de hacer el diagnóstico, su contundente alcance hace necesario realizarlo, pero con procesos, técnicas, instrumentos y herramientas que den seguridad de la calidad de los datos.

\section{Conclusiones}

El primero de los puntos a tratar en estas conclusiones es logro del objetivo, el alcance de esta investigación es descriptivo llegando al establecimiento de inferencias entre los docentes que participaron, de ahí es que se considera haber cumplido a totalidad el propósito de estudiar las necesidades de capacitación y las competencias docentes de educación media superior en la Universidad Virtual del Estado de Guanajuato.

Esto nos remite a la extensa cobertura que tiene la Universidad Virtual del Estado de Guanajuato, al poseer una modalidad en línea permite llegar a diversas personas, ya sean administrativos, docentes, profesores, estudiantes, etc. Por otra parte, el Departamento de Formación y Desarrollo Docente se encarga de las capacitaciones de los docentes de modalidad 
presencial, con la finalidad de establecer procesos claros y tener efectividad al momento de lanzar los cursos o talleres, además de generar información de calidad para poder atender objetivamente las demandas de los docentes.

Los retos que enfrenta corresponden a cumplir de forma puntual con un plan de capacitación que realmente se ajuste a las necesidades del personal docente, mediante la aplicación de procesos con rigurosidad científica y difundir por canales formales y serios los resultados.

Dichos resultados deducibles de esta muestra orientan a la comunidad científica a la elaboración de estrategias y toma de decisiones al momento de pretender capacitar; hace evidente en qué grupos de docentes es preferible dar atenciones de capacitación y cuáles son las demandas de capacitación del personal docente, además de mostrar una ruta en la detección de necesidades de capacitación.

\section{Referencias}

Asún, R., Zúñiga, C., \& Ayala, M. C. (2013). La formación por competencias y los estudiantes: confluencias y divergencias en la construcción del docente ideal. Revista Calidad en la Educación, (38), 277-304. https://dx.doi.org/10.4067/S0718-45652013000100008

Bermúdez, L. A. (2015). Capacitación: una herramienta de fortalecimiento de las pymes. InterSedes: Revista de las Sedes Regionales, 16(33), 1-25.

Castillo, V., Yahuita, J., \& Garabito, R. (2006). Estrategias docentes para un aprendizaje significativo. Cuadernos Hospital de Clínicas, 51(1), 96-101.

Camargo-Escobar, I. M., \& Pardo-Adames, C. (2008). Competencias docentes de profesores de pregrado: diseño y validación de un instrumento de evaluación. Competencias docentes de 
profesores de pregrado: diseño y validación de un instrumento de evaluación. Universitas Psychologica, 7(2), 441-457.

Castro-Granados, M. A., Medina-Almeida, C., \& Glasserman, L. D. (2017). Fortalecimiento de las competencias docentes para atender el cyberbullying en una secundaria pública colombiana. CPU-e Revista de Investigación Educativa, (24), 199-223.

Cota, J. A., \& Rivera, J. L. (2017). La capacitación como herramienta efectiva para mejorar el desempeño de los empleados de una cadena de zapaterías de ciudad obregón, sonora. Técnica Administrativa, 16(2). http://www.cyta.com.ar/ta/art_ficha.php?id=160203

Cruz, O. F., \& Surdez, E. G. (2018). Detección de necesidades de capacitación: actividad para desarrollar el capital humano. Revista Global de Negocios, 6(3), 57-68.

García, B., Luna, E., Ponce, S., Cisneros-Cohernour, E. J., Cordero, G., \& Espinosa, Y. (2018). Las competencias docentes en entornos virtuales: un modelo para su evaluación. RIED. Revista Iberoamericana de Educación a Distancia, 21(1), 343-365. http://dx.doi.org/10.5944/ried.21.1.18816

González-Verde, A., \& Muñiz-Izquierdo, N. M. (2016). Procedimiento para el diagnóstico y proyección de la formación por competencias. Ingeniería Industrial, 37(3), 266-277.

Guiñazú, G. (2004). Capacitación efectiva en la empresa. Invenio, 7(12), 103-116.

Guzmán, I., \& Marín, R. (2011). La competencia y las competencias docentes: reflexiones sobre el concepto y la evaluación. Revista Electrónica Interuniversitaria de Formación del Profesorado, 14(1), 151-163.

Guzmán, I., Marín, R., Zesati, G. I., \& Breach, R. M. (2012). Desarrollar y evaluar competencias docentes: estrategias para una práctica reflexiva. Voces y Silencios: Revista Latinoamericana de Educación, 3(1), 22-40.

Herrera, C. A., Niklitschek I., Pizarro M., Solís, N., Olivos, T., Rojas, V., Etcheberry, L., Rivera, H., Muñoz, E., Bitran, M., Padilla, O., \& Riquelme, A. (2013). Identificación de las 
necesidades de capacitación docente de los jefes de programa de especialización médica. Revista Médica de Chile, 141(9), 1117-1125.

Lay, M. T., Suárez, J., Zamora, M. (2005). Modelo para gestionar la capacitación de directivos y reservas en la empresa ganadera cubana. I. Diagnóstico empresarial y del proceso de capacitación. Pastos y Forrajes, 28(3), 253-263.

López, J. M., \& Sarabia, L. Y. (2016). Sistematización de un modelo de capacitación del personal administrativo de apoyo de la universidad centroccidental "Lisandro Alvarado" (UCLA). Revista Científica Teorías, Enfoques y Aplicaciones en las Ciencias Sociales, 9(19), 55-70.

López, R., Reyes, Y., \& Molina, A. T. (2017). Evaluación del impacto de la capacitación en directivos de instalaciones turísticas. Ciencia, Docencia y Tecnología, 28(54), 130-149.

Maya-Ampudia, C. C., Ángeles-Zavala, O., Camarena-Olmedo, J. A. Diagnóstico de necesidades de educación continua mediante un método grupal y un método individual. Investigación en Educación Médica, 3(12), 177-186. https://doi.org/10.1016/S2007-5057(14)70933-0

Méndez, E. (2002). Importancia de la detección de necesidades de capacitación y formación de la CCSS. Revistas de Ciencias Administrativas y Financieras de la Seguridad Social, 10(2), 65-72.

Méndez, E. (2004). El Diagnóstico de Necesidades de Capacitación es un asunto local. Revistas de Ciencias Administrativas y Financieras de la Seguridad Social, 12(1), 25-33.

Martínez-Miguel, E., Solano, M. C., García-Carpintero, E., \& Manso, C. (2018). Impacto de la evaluación de competencias en la calidad del aprendizaje: percepción de discentes y docentes de grado en enfermería. Enfermería Global, 17(50), 400-429.

Polo, M. (2003). El DENEPER: Una Metodología para detectar necesidades de perfeccionamiento docente. Revista de Pedagogía, 24(71), 385-415.

Puente, A., Acosta, H. G., Beltrán, F. J., \& Torres, I. A. (2002). Detección de necesidades de capacitación de personal en una organización educativa. Enseñanza e Investigación en Psicología, 7(2), 257-268. 
Rangel, A. (2015). Competencias docentes digitales: propuesta de un perfil. Pixel-Bit. Revista de Medios y Educación, (46), 235-248. http://dx.doi.org/10.12795/pixelbit.2015.i46.15

Revilla, N. V., Acosta, I. C., \& Marval, E. V. (2009). Necesidades de entrenamiento del personal basado en el enfoque de competencias: Estudio de un caso. Revista Venezolana de Gerencia, 14(46), 195-214.

Ruíz, S., Baca, M. E., \& Gutiérrez, J. M. (febrero, 2011). Detección de necesidades de capacitación. Caso: Auditoría Superior de la Federación. Trabajo presentado en la conferencia del XIV Congreso internacional sobre innovaciones en docencia e investigación en ciencias económico administrativas, Guanajuato.

Salas, R. S. (2003). La identificación de necesidades de aprendizaje. Educación Médica Superior, 17(1), 25-38.

Sapién, A. L., Piñón, L. C., Gutiérrez, M. C. (2014). Capacitación en la empresa mexicana: un estudio de formación en el trabajo. Civilizar, 14(27), 123-134.

Secretaría de Educación Pública, (2019). Acuerdo número 447 por el que se establecen las competencias docentes para quienes impartan educación media superior en la modalidad escolarizada.

Secretaría de Educación Pública, (2019). Marco para la excelencia en la enseñanza y la gestión escolar en la Educación Media Superior: perfiles profesionales, criterios e indicadores para docentes, técnicos docentes y personal con funciones de dirección y de supervisión.

Tejada, J. (2009). Competencias docentes. Profesorado. Revista de Currículum y Formación de Profesorado, 13(2), 1-15.

Torres, A. D., Badillo, M., Valentin, N. O., \& Ramírez, E. T. (2014). Las competencias docentes: el desafío de la educación superior. Innovación Educativa, 14(66), 129-145.

Velázquez, A. E., \& Peinado, J. J. (2010). Propuesta de un programa de capacitación para el personal de apoyo y asistencia a la educación del Instituto Politécnico Nacional: un estudio 
de caso, el centro de investigación e innovación tecnológica. Investigación Administrativa, 39(106), 83-96.

Vergel, M., Parra, H. M., \& Martínez, J. J. (2013). Metodología para elaborar planes de capacitación en instituciones de educación superior. Revista Logos, Ciencia \& Tecnología, 5(1), 205-213.

Villarroel, V. A., \& Bruna, D. V. (2017). Competencias pedagógicas que caracterizan a un docente universitario de excelencia: un estudio de caso que incorpora la perspectiva de docentes y estudiantes. Formación Universitaria, 10(4), 75-96. http://dx.doi.org/10.4067/S071850062017000400008

Werther. W., \& Davis. K. (2008). Capacitación y desarrollo. En Administración de recursos humanos, el capital humano de las empresas. (pp. 252 - 280). McGraw-Hill.

https://revistainvestigacionacademicasinfrontera.unison.mx/index.php/RDIASF/article/view/382

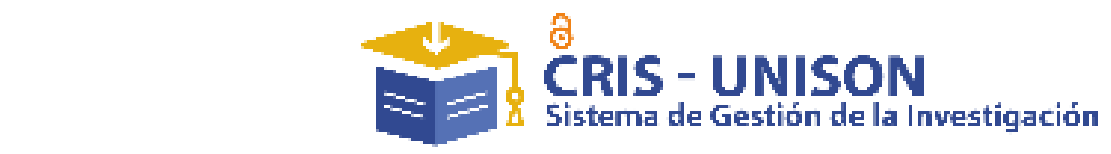

- Dialnet

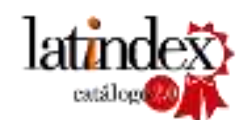

If Inifactor

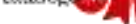
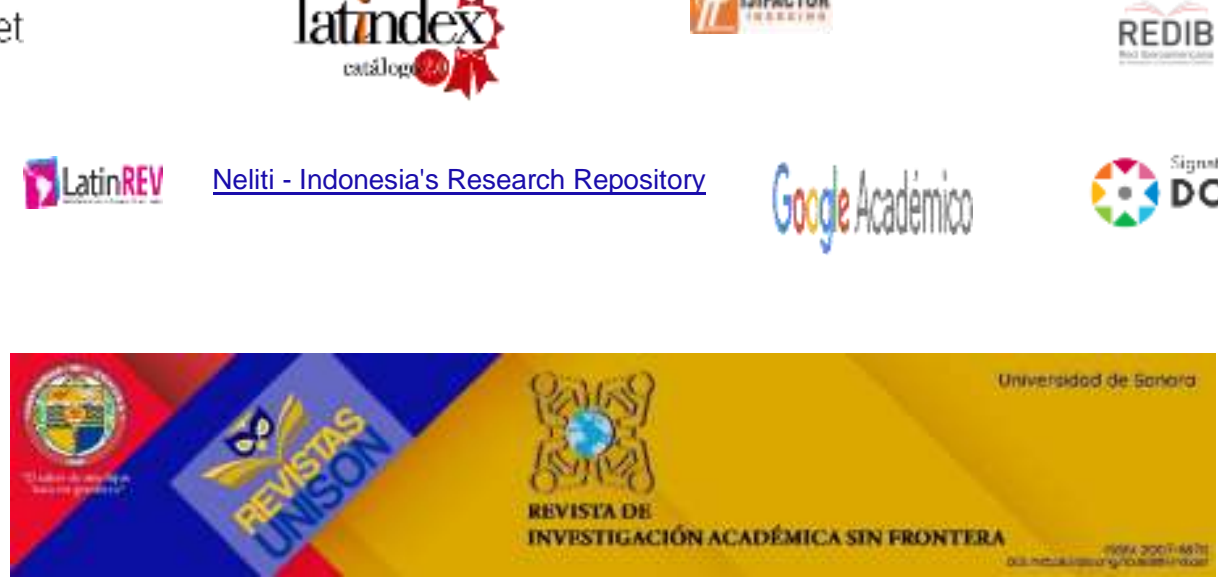